have had the advantage of hearing the profession's views, which were stated by a representative deputation which waited upon the Minister for Co-ordination of Defence last November. The Government was then urged first to provide some machinery whereby the demands of the Navy, Army, Air Force, and civilian population for medical personnel could be co-ordinated, and, secondly, that the Central Emergency Committee and the Scottish Emergency Committee set up by the British Medical Association at the request of the Government should be regarded as the sole sources through which these demands should be met. We have gone a long way towards adopting these suggestions. As regards the first, we are ascertaining so far as possible the requirements of the Service Departments in medical personnel, so that they can be related to the demands for the civilian services of the profession. Further, the responsibility has been placed upon the Ministry of Health in England and the Department of Health in Scotland, not only of making plans for the hospital treatment of civilian air raid casualties but also of organizing the first-aid posts and the ambulance services, which must obviously play a most important part in any casualty service. The ordinary peace-time responsibility for seeing that the public health and insurance medical services are maintained remains, of course, with these Departments. Thus so far as the civilian population is concerned much has been done to combine under one single direction the whole of the medical arrangements for meeting an emergency.

As regards the machinery for meeting these demands, we have available in the Central Emergency Committee and the Scottish Emergency Committee set up by the British Medical Association two central and expert advisory bodies. These committees are, as you will be aware, representative of the whole of the profession: But if the committees' advice is to be sound it must necessarily take account of the local conditions affecting the profession. I am glad, therefore, that the British Medical Association has recently advised each of its Divisions to call a meeting at once of the whole profession for the purpose of setting up a local emergency committee, which will also be representative of the profession as a whole in the area, and will thus be able to advise the Central Emergency Committee on questions of personnel from the local point of view.

Apart, however, from a certain amount of earmarking we cannot fix in advance the capacity in which each individual practitioner can best render service in an emergency. We shall, however, do our best in this direction, and Mr. Colville and I are having a meeting with the English and Scottish Central Emergency Committees and representatives of the local emergency committees on February 15 to see in how much detail we can develop our plans for the allocation of medical personnel.

There is, however, one piece of advice which I should like to emphasize, and that is that every practitioner, except, of course, those having obligations to the Forces, should remain at his peace-time post, even though an emergency should arise, until he is asked to do something different. I hope he may rest assured that we are doing everything we can in consultation with the duly accredited representatives of the profession, both local and central, so to organize the personnel available as to ensure that it is used to the best advantage.

To sum up, life and death are the raw materials of the State, and those dealing with life and death must always and inevitably be under some compulsory con- nexion with the State, call it organization or what you will, both in peace and in war. This connexion, as I have shown, is very old and is still actively developing almost every hour to-day. Since that is so the relations between both the medical profession and the State must be as cordial and as personal as can be accomplished. That is why I have taken this long road to see you and speak to you to-night.

\section{THE TREATMENT OF TRICHINOSIS}

\section{A REVIEW OF METHODS}

\section{BY}

VERNON D. van SOMEREN, B.Sc.

Research Student, Department of Parasitology, London School of Hygiene and Tropical Medicine

In countries where trichinosis appears still to be widely prevalent, as in the U.S.A., the majority of cases generally are of a symptomless subclinical type, and as such are discovered only by post-mortem examination or by use of the Bachman intradermal test. Naturally such cases do not come within the purview of the general practitioner, but every now and then the disease assumes epidemic proportions of a more or less severe character, while severe sporadic cases are also not uncommon where meat inspection and proper cooking of pork are not rigidly practised.

Since such cases are seldom diagnosed during the incubation period, but only during the acute migratory phases, immediate treatment is essential to prevent the onset of graver complications in an already debilitated patient, and the fact that a wide variety of methods have been tried is in itself indicative of the present experimental stage in the treatment of the disease. Such experimentation may possibly have unfortunate results; hence it seems advisable to summarize the available knowledge concerning treatment and indicate the more hopeful lines of progress. It is proposed to deal only with methods specifically directed against the parasite in the body, and not with the purely symptomatic clinical treatment: this latter, however, may briefly be summarized.

Trichinosis being a disease inducing a febrile state, a general fever regimen should be followed, and since the symptoms are those of a more or less profound toxaemia, all necessary supportive measures should bè provided, followed by a prolonged convalescence period. The bowels should be kept open and alkalinized, and special attention paid to the kidneys. Heart and respiratory stimulants should be provided, and sterile saline given to counteract dehydration (Craig and Faust, 1937). Muscular pains and fever associated with the late migratory and early encystment stages of the disease should be relieved by sedatives and antipyretics, though it is of note that salicylates are of little use in these respects (Herzberg and Vitenson, 1930). Possible allergic symptoms may be controlled by the intramuscular injection of 0.5 to $1 \mathrm{c.cm}$. of adrenaline, as often as the somewhat transient improvement might demand; while meningismus, a not uncommon feature of the syndrome, might be ameliorated by lumbar puncture (Fantus, 1935). The patient should be kept on a high-calorie diet (McDonald and Waddell, 1929).

While measures directed to support and perhaps increase the natural resistance of the host to the parasite are the most valuable in the present state of knowledge, 
it is not surprising that a variety of methods have been tried to secure destruction of the parasite in the body, with varying results. Trichinella parasitizes the host in two distinct phases. There is at first an intestinal phase, during which most of the ingested larvae mature and the resulting adults live and reproduce in the mucosa and submucosa of the intestines-a phase lasting several weeks in man; this phase gives rise to a subsequent and partly coexistent somatic phase when the larvae released by the adults in the intestines pass via the lymphatics and blood stream to all parts of the body, finally becoming localized in the voluntary muscles, where they encyst, and, after several years, calcify and die if not reingested by another host. The existence of these two phases complicates the therapy of the disease; the localized intestinal phase appears in general to be more subject to destructive measures, although the habitat of the adults deep in the mucosa and submucosa and surrounded by mucus renders difficult the action of any therapeutic measure directed against them. Another difficulty also arises from the fact that, while most of the adults are located in the posterior part of the ileum, a certain number also live in the large intestine and caecum, especially in massive infections (Roth, 1938) ; consequently any drug which may be of use against this stage must not be entirely absorbed in the small intestine, but must be capable of acting also in the caecum and colon.

The somatic phase, which is at first widespread and then localized in voluntary muscles, is far less amenable to any form of treatment, and, unfortunately, diagnostic measures are such that the somatic phase is usually well established by the time specific diagnosis is possible. While this phase is immediately the most dangerous, it is advisable still to try measures which may be of use against adults only, as these are potentially dangerous throughout the whole of their reproductive life, and any attempt to lessen the severity of the muscle invasion is a rational therapy. For the same reason any substance which may be lethal to migrating larvae should also be lethal to intestinal adults, but the possibility that the various stages differ in their susceptibility to the same drug should be borne in mind (van Someren, 1938).

Methods which have been tried can conveniently be classified into (1) mechanical, (2) biological, (3) chemotherapeutic, and (4) chemico-biological methods, but it should be stated at the outset that no experimentally confirmed specific cure for any or all of the phases has yet been discovered.

\section{Mechanical Treatment}

Mechanical dislodgment or destruction of the various stages of Trichinella in the body has been attempted in two ways, neither of which is more than partially successful.

(a) Radium Irradiation.-Tyzzer and Honiej (1917) have investigated the effect of radium irradiation, external and internal, on trichinosis of the rat. Irradiation from the surface of the abdomen does not destroy adult trichinae in the intestine, but if started on the second day after infection it exerts a retarding effect on the further development of the larvae, 30 per cent. being immature when compared at corresponding periods with controls. Internal irradiation from the intestine by radon seeds, however, does not prevent development. Encysted larvae are rendered non-infective (not necessarily killed) by $6.6 \mathrm{mc}$. emanation at a distance of $1.5 \mathrm{~mm}$. through $0.1 \mathrm{~mm}$. steel filtration for thirty minutes. Obviously these results have little practical application, since, unless the initial infective dose is large enough to produce gastrointestinal symptoms (whith are not in themselves specific), the disease cannot be diagnosed sufficiently early, even by the Bachman skin test, to enable irradiation to be of any use for retardation purposes, because the larvae become sexually mature in the short space of five to seven days or less; nor would such retardation prevent the ultimate development of a migratory phase. Similarly also the treatment of encysted larvae to render them noninfective is of little use in the human subject. The acute stages of the disease have passed by the time the larvae have encysted, and the short distance of irradiation necessary precludes the possibility of treating the whole body.

(b) Purgation.-Drastic catharsis, especially in the early stages, but even up to six weeks after infection, has been recommended in the hope that adult worms may be swept out. McDonald and Waddell (1929) have found it effective to give 2 grains of calomel, followed by high colonic irrigation ; and Fantus (1935) advises 0.2 gramme ( 3 grains) of calomel, followed in six to eight hours by 30 grammes (450 grains) of magnesium sulphate, and this by colon flushing several hours later, the treatment being repeated for several days. While such treatment may be effective in the very early intestinal stages, its efficacy after larviposition has started is very doubtful, in view of the deep-seated habitat of the adults during this stage, and the difficulty of an early diagnosis is again a drawback. Flushings and enemas per se are of no practical advantage, as they cannot reach those adults in the ileum. The administration of glycerin has been suggested for its hygroscopic properties, but Grove (1925) and Van Cott and Lintz (1914) have doubted the effectiveness of this method, and again the habitat of the adults would seem to make its use of little value.

\section{Biological Treatment}

Biological methods of treatment consist in the administration of serums prepared from actively trichinosed or convalescent cases. Such serum treatment has been very highly recommended by Salzer $(1916,1917)$, who claimed that serum from convalescent human cases removed persistent eosinophilia in forty-eight hours, while normal serum, salvarsanized serum, and saline have no therapeutic value; that convalescent serum gives an almost complete prophylaxis if infection takes place later than twenty-four hours after injection; that convalescent serum mixed with infected meat prevented infection taking place ; that in two cases in man such immune serum was curative, causing the temperature to fall in six hours and become normal in forty-eight hours, eosinophilia being reduced in six hours; and that twenty-four rabbits, experimentally infected, were cured in twenty-four hours by injection of immune serum.

These extensive claims for the use of serum were experimentally investigated by Schwartz (1917), who could find no support for any of Salzer's contentions. In view of such conflicting results the use of serum was reinvestigated experimentally by Hall and Wigdor (1918), who agreed with Schwartz in his findings that convalescent serum injections, or such serum mixed with infected meat, does not inhibit development of trichinosis, but concluded that such serum may be of decided value in combating the toxic features of the disease.

This question of the development of immunity in trichinosis and the use of immune serum has been investigated by several other workers. McCoy (1935) found that immunity against the intestinal stages of trichinosis in rats could be produced by intraperitoneal injections of living larvae, or 
heat-killed, dried, and powdered larvae. Trawinski (1935) concluded that serum taken from trichinosed rabbits between twenty-five and thirty-five days after infection possessed antitoxic but not anti-parasitic properties, and recommended three subcutaneous injections of $1 \mathrm{c.cm}$. as a specific therapy. Bachman and González (1936) determined that trichinous antiserum from rabbits conferred no immunity in rats when fed by mouth, nor was convalescent serum from pigs of any use for the same purpose. Dried trichina powder, or intraperitoneal suspensions of dried powder in Coca's solution, similarly were of no use for immunization purposes.

Since the technique of experimentation has differed in such studies, Culbertson and Kaplan (1937, 1938) have investigated the problem, basing their conclusions on actual counts of intestinal adults and larvae in the muscles in relation to known numbers of larvae used for a given infection ; obviously a more reliable method, for example, than the study of the longevity of infected animals used as a basis by Hall and Wigdor (1918). Culbertson and Kaplan found that mice were partially protected against Trichinella by injection with anti-Trichinella rabbit serum, a smaller proportion of infected animals dying, and those remaining having fewer larvae in the muscles than controls. Such immune serum appears to act specifically against the larvae maturing to adults in the intestine, 17.6 per cent. of the total of larvae fed maturing in treated mice, as against 36 per cent. in mice treated with normal serum and 37.1 per cent. in untreated controls. The ratio of larvae encysted in the muscles to the number of larvae fed was 44.0 to 1 in immunized mice as against 69.9 to 1 in untreated controls. It seems, therefore, that immune serum does not act on larvae migrating to or in the muscles, since that percentage of infective larvae has already been inhibited in the intestine in treated mice. These authors conclude that the antibody produced is blood-borne and is a generalized response to infection, but that passive immunization has its action locally on ingested larvae developing to adults in the intestine, though such immunity is not complete. It is of interest to note that the natural age immunity shown by dogs is also of this nature, being directed only against development of adults in the intestine (Matoff, 1937). There is some evidence that previous infection may confer partial immunity against reinfection (McCoy, 1931).

Apart from the question whether or not man could be immunized against trichinosis by any of these methods, and how long such immunity would last, the wholesale immunization of groups of persons would be of little practical value, since prophylaxis can be much more efficiently and economically carried out by rigid meat inspection, and more especially by proper cooking of pork and pork products and other possibly infected meat, such as bear meat.

Concerning the value of convalescent antiserum in treatment, the balance of opinion favours the view that serum inhibits parasite development in the early phases of the disease, and is antitoxic but not parasiticidal in the later phases: As to its inhibitory properties in the early phase of trichinosis one is again faced with the disadvantage of not being able to form a diagnosis early enough-that is, before the larvae have matured in the intestine. The incubation period in man appears generally to be twelve to thirteen days (Ferenbaugh et al., 1938), and the Bachman intradermal test-the most reliable form of early diagnosis, since eosinophilia and other features are not evident until larviposition has begun-does not become positive until about sixteen days after infection at the earliest in man (Augustine and Theiler, 1932; Maternowska, 1933). Such a limited period of time during which serum would be of use for this purpose would make it of no significance in treatment unless methods of early diagnosis could be improved.

There remains the fact that serum may be of distinct advantage in controlling the toxaemia of the later stages, could it be obtained. In countries such as Great Britain, where subclinical trichinosis is uncommon and clinical trichinosis rare (van Someren, 1937), the commercial production of serum would be unnecessary; but in other countries, such as the U.S.A., where clinical trichinosis is not uncommon, the commercial production of serum might well be tried. The economic production of large quantities might, however, prove a difficulty, since Trichinella will not develop readily, if at all, in herbivores, such as the horse, used for large-scale production of other serums and antitoxins. More research concerning the possibilities of the use of serum and its keeping qualities is required, and beyond Salzer's contribution no clinical trials of serum have been made.

\section{Chemotherapeutic Methods}

A wide variety of drugs have been tried experimentally and clinically in attempts to secure destruction of the parasite in the body, with limited success. These may conveniently be classified into: (a) anthelmintics; (b) antiseptics, disinfectants, and related substances; (c) organic arsenicals and similar substances; and (d) miscellaneous substances. In general, the doses which have been used are not given below, since these have all been in accordance with recognized clinical practice, correspondingly reduced for experimental trials in animals. The total doses used in intravenous therapy have usually been less than those recommended for diseases for which the drugs are specific.

ANTHELMINTICS

It is natural that most of the recognized anthelmintics should have been tried in trichinosis, but the fact that trichinae exist in a tissue phase as well as an intestinal limits the use of those anthelmintics which are only partly or not at all absorbed from the intestine, unless such can be administered safely by the subcutaneous or intravenous route.

Thymol, which has been used extensively with success against hookworm, particularly Necator, but has been stated to have only a slight action on other nematodes (Ascaris) (Clark, 1929), has been recommended as a specific for trichinosis; it has been given per os for the intestinal phases and as a sterile suspension in olive oil for the migratory phases without any toxic effects. Booth et al. (1916), Wenderoth (1917), Kahn (1917), Munk (1917), Gruber (1926), Seifert (1929), and Herzberg and Vitenson (1930) have all advocated the use of thymol, or noticed a curative effect in reduction of eosinophilia and other symptoms in man after use. Grove (1925), however, has stated that thymol appears to be of little value, and Baudet (1931) has investigated its effect in experimental rats and found that oral or subcutaneous doses of thymol have no trichinicidal action, nor can thymol hinder the development of trichinosis even when treatment is started one day after infection.

Cariasept, a complex commercial preparation of thymol, has been found by Sprehn (1931) to have an anthelmintic action on both cestodes and nematodes. This substance was also investigated experimentally by Baudet (1931), and was shown to have a rather more favourable effect than thymol, subcutaneous injections of suspensions in olive oil or gum arabic being lethal to adult trichinae; no complete destruction of adults, however, was observed, and since one adult female trichina may give rise to 200 to 300 larvae the use of carvasept could be ameliorative only. 
Butolan, a commercial preparation of the carbamic acid ester of $p$-oxy-diphenyl-methane, the active anthelmintic principle of which is $p$-benzylphenol (Schulemann, 1920), was tried in experimental trichinosis of the rat by van Someren (1938) and shown to have a lethal effect on a considerable proportion of intestinal adults with total doses of 1.5 grammes (22 $\frac{1}{2}$ grains) or over. It does not, however, have any effect on migrating or encysted larvae per os; but in spite of this the substance appears worthy of a clinical trial in human trichinosis, since adults live for several weeks in man and any attempt to lessen the severity of the muscle invasion by destruction of reproductive adults forms a rational therapy. Butolan appears of greater efficacy in trichinosis than the related thymol preparations, and the only contraindications to its use would be evidence of severe kidney damage. It is easy to administer and is relatively non-toxic.

Beta-naphthol is said to have a lethal effect on adults (Chopra and Chandler, 1928), but has not been investigated experimentally. Santonin, aspidium, and tetrachlorethylene have all been stated by Grove (1925), Hardy (1927), and Sogemeier (1938) to be of little use. Gentian-violet, which has been found by Faust (1929) to have markedly clonorchicidal properties, and is also specific for Strongyloides stercoralis, a nematode having the same deep-seated habitat in the adult stage in the crypts of the mucosa (Craig and Faust, 1937), has been tried intravenously in experimental trichinosis of the rabbit by Miller et al. (1932), but has no effective action.

ANTISEPTICS. DISINFECTANTS, AND RELATED SUBSTANCES

Neutroflavine injections were observed by Africa and Lucker (1931) to reduce the degree of muscle invasion in experimental trichinosis, but this was not substantiated by Miller et al. (1932), who found intravenous injections of acriflavine base and the related rivanol to be of no value in experimental trichinosis. Iodine, in the form of Lugol's solution or the salt potassium iodide, can be shown to have a powerful lethal effect on Trichinella larvae and adults in vitro, but intravenous injections of Lugol's solution or intraperitoneal injections of sodium iodide were found by Miller et al. (1932) to be of no value in treatment. Metaphen, a bactericidal organic mercury compound, was similarly found by these authors to be ineffective intravenously. Hexyl-resorcinol is stated by Sogemeier (1938) to be of no value. Cuprosal, a coppercontaining antiseptic, was found to be ineffective when injected (Fleischmann, 1930). Sulphanilamide, the powerful dye derivative which is now used extensively in the treatment of bacterial infections with the coccal group, has been tried in experimental trichinosis of the rat by McCoy (1938), and shown to be ineffective in controlling the disease.

\section{ORGANIC ARSENICALS AND SIMILAR SUBSTANCES}

In view of the pronounced parasiticidal properties shown by the organic arsenicals and antimony salts in the treatment of spirochaetal, trypanosomal, and certain helminthic diseases with widespread tissue infection, many attempts have been made to treat trichinosis with these drugs.

Neoarsphenamine (Neosalvarsan) - McNerthney and McNerthney (1916), Algora y Nieto (1924), and Martin (1936) have all reported beneficial results in human cases of trichinosis after intravenous administration of neosalvarsan, the drug appearing to alleviate the symptoms and forming a rational therapy during the migratory phases; but Van Cott and Lintz (1914) and Fleischmann (1930) found the drug useless in human cases, while Miller et al. (1932) showed that it was ineffective in destroying the parasites in experimental trichinosis of the rabbit.

Silver salvarsan was found by Ragany (1935) to be effective in eight cases of human trichinosis, but no experimental trials of this form of salvarsan have been made. Atoxyl and arsacetin are stated by Sogemeier (1938) to be of no value. Antimony oxide was tried early in the migratory phase of trichinosis in man by Hardy (1927), but no definite effect was observed.
Potassio-tartrate of antimony (tartar emetic) was considered by Grove (1925) to be of some use in trichinosis before localization of the larvae, and Caronia (1937) reports a case cured after treatment with antimony and urges its more extensive use. Hassin and Diamond (1926), however, found it of no use in a human case with nervous involvement, and Miller et al. (1932) were unsuccessful in treating experimental trichinosis of the rabbit with this drug.

\section{MISCELLANEOUS SUBSTANCES}

Causyth, a commercial preparation of the cyclohexatrienepyridine-sulphonate of a pyrazolone derivative $\left(\mathrm{C}_{22} \mathrm{H}_{24} \mathrm{~N}_{4} \mathrm{O}_{5} \mathrm{~S}\right)$, claimed to be of therapeutic value in certain febrile and infectious illnesses, was stated by Weltmann (1931) to have a favourable effect in a human case of trichinosis, 6 grammes (90 grains) of causyth in $50 \mathrm{c.cm}$. of water being given per rectum. Baudet (1935) investigated this substance experimentally and found that causyth per os did not prevent development of either intestinal or muscular trichinosis, although given two days after infection. The favourable effect in Weltmann's case was considered by Baudet to be due to an analgesic and antipyretic action of the drug. Novasurol, a mercurial diuretic, was reported by Jochweds and Pekielis (Chükri, 1930) to be of use in human trichinosis; but Chükri (1930) found it had no lethal effect on any stages of Trichinella in the rat. Lecithin and copper, in a preparation known as lekutylpillen, was found to be of no value by Fleischmann (1930) when administered per os in human trichinosis.

Alcohol.-The liberal use of alcohol in the form of cognac was advocated by earlier writers for the treatment of trichinosis. Pierce and McNaught (1937) showed, in vitro, that concentrations of alcohol as low as 9 per cent. interfered with excystment of larvae, but this was possibly due to destruction of the proteolytic activity of the gastric enzymes by the alcohol. A concentration of as much as 25 per cent. alcohol has little direct action on predigested larvae, which can remain alive in this percentage for more than six hours-and this is a strength that cannot be maintained in the stomach for such a long period. Alcohol is therefore of no therapeutic value if the larvae have already been freed.

I have been unable to obtain the paper by Greenwood (1935) on the chemotherapy of experimental trichinosis.

\section{Discussion on Chemotherapeutic Methods}

This review of chemotherapeutic methods brings into evidence one striking fact-that many drugs which have been claimed to have a favourable or curative effect in man have been quite ineffective when tested experimentally. There is no reason to suppose that the physiology and pathology of Trichinella and trichinosis in experimental animals are different from those in man, and the experimental test showing the actual destruction of the parasite must remain the decisive factor in determining the efficacy of the drug for the purpose.

In not one case in which favourable results have been reported in man has actual destruction of the parasites been shown, nor have such cases been controlled in any way; while it should be noted that in most uncomplicated human cases the prognosis is favourable and recovery assured, the death rate being low except in epidemics. The favourable effects in all cases have been amelioration of the symptoms, which may be due to a purely symptomatic action of the drug; in some instances the disappearance of eosinophilia has been taken as demonstrative of a curative result. Eosinophilia appears to be a response of the body to the introduction of certain foreign proteins or non-bacterial toxins, particularly of an animal type, and certain chemicals, such as camphor, also are capable of inducing eosinophilia. It has been shown by Banerji (1933) that cusinophilia is dependent on the acidbase equilibrium of the blood as well, and it is a sensitive 
reaction which may easily be destroyed by hyperinfection with pyogenic organisms (Spink, 1934). It is not inconceivable, therefore, that the introduction of drugs into the blood stream may also in some way cause the reduction of eosinophilia, and this alone cannot be taken as indicative of a favourable effect. Trichinella is usually lethal to hosts which fail to respond by an appreciable eosinophilia, and possible interference with this defence reaction is to be deprecated.

Miller et al. (1932), in their critical study of intravenous therapy in trichinosis, have shown that not only are all the drugs which have been tried useless but that such treatment may be dangerous during the course of the disease. Severe trichinosis produces marked toxic degenerative changes in the kidney, which may well hinder the proper excretion of drugs, particularly those containing the heavy metals. It is also possible, should a lethal drug be discovered, that the migratory larvae, which are of a relatively large size, might, unless phagocytosed rapidly, form fatal emboli, since they are widespread in the body fluids and organs before localization in the voluntary muscles. The possibility of anaphylactic phenomena following any destruction of the parasite in the body should also be borne in mind.

\section{Chemico-biological Methods}

For treatment in the later stages of the disease a rather more hopeful line of progress has been experimented with. As is well known, the larvae on reaching voluntary muscles become surrounded by an adventitious fibrous capsule which after several years (in man) becomes calcified, such calcification being followed by death of the larva within the cyst. Until such calcification and death occurs the host may still show toxic symptoms, particularly in the early encystment stage. Use has therefore been made of the parathyroid-vitamin-D-calcium complex to secure forced calcification and death of the parasite within a much shorter space of time than is natural.

Irradiated Ergosterol.-Von Brand et al. $(1932,1933)$ have succeeded in producing cyst calcification experimentally two to three months after infection by daily administration of irradiated ergosterol; and Wantland (1934), using 30 to 60 drops of irradiated ergosterol every other day, together with a dose of 0.5 gramme to 2 grammes of calcium lactate, has shown that calcification of cysts in white rabbits may be obtained in less than six weeks, instead of the normal seven to eight months, the onset of calcification being observed in the early part of the fourth week following infection. In white rats (Wantland, 1936, 1938) 40 to 50 drops of irradiated ergosterol alone daily from the fifty-fourth day after infection produce calcification in three months. Larger doses of ergosterol are toxic, and the calcification in rats is not accelerated so much as in rabbits. The results have shown that calcification usually results in death of the larvae, the calcification being subsequently reabsorbed. In cats (Wantland, 1936) doses of 40 to 70 drops of irradiated ergosterol (containing 10,000 U.S.P. units of vitamin D per gramme), together with ordinary milk, given daily from the third day after infection, produce slight calcification as early as the thirtieth day after infection, and medium to marked calcification fifty to fifty-five days after infection. Toxic symptoms were, however, noticed with these doses of ergosterol.

Irradiated milk produces no early calcification in cysts in cats, though the cysts become slightly thicker at the poles than in controls (Wantland, 1936).

Parathormone (subcutaneously) plus calcium lactate does not accelerate calcification in trichinosed white rabbits, although given at various times in various doses (Wantland, 1935). In rats, however, von Brand et al. $(1937,1938)$ have shown that while no calcification is produced fourteen to seventeen days after infection by subcutaneous injections of parathormone, calcification may occur in two days in cysts four weeks to four months old after use of the hormone. Such calcification, however, does not proceed faster than calcification of other host tissues following injection of parathormone, and in fact intensive use of parathormone for this purpose usually results in death of the host. These authors conclude that any attempt to procure forced calcification with parathormone, irradiated ergosterol, or other agents is futile with the usual human doses, and dangerous if used in doses of a calcifying level; and they further state that calcification does not kill the larvae, but that the larvae themselves calcify after natural death.

Despite this warning concerning the use of the method, further work might show that it has therapeutic possibilities. Manifestly it cannot be used as a primary measure during the acute migratory stages, but any attempt to secure the host against possible toxic symptoms in the early encystment stage would seem to be a rational secondary treatment. Unfortunately experiments with laboratory animals on these lines cannot be taken as indicative of measures that could be applied to man, since different animals vary in their reactions to these substances, and the reactions of man himself must be studied.

The method actually has had a clinical trial in an outbreak of trichinosis. Hall (1937) treated his cases with calcium gluconate, and supplied vitamin $\mathbf{D}$ in the form of halibut-liver oil and viosterol; he reports that "the apparent effect of this therapy on the fever encourages us to believe that it may have brought about a shortening of the encystment stage." These results are encouraging, and a more extensive clinical trial should be made. Fantus (1935) has suggested that parathyroid extract be used, 20 to 40 units being given every twelve hours for not more than ten days, supplemented by a course of calcium lactate and lactose. Parathormone, for this purpose, has not yet had a clinical trial, but it would appear preferable to use ergosterol or some similar vitamin-D-containing substance as the calcification-induction agent, supplemented by a supply of calcium in the form of a bland organic salt, rather than parathormone, since the latter may produce a dangerous hypercalcaemia. It would seem advisable to begin any such course of treatment about the fourth week of the disease, when early encystment starts.

Recently Sogemeier (1938) has reported very favourably on another form of calcium therapy which appears to be of use during the acute migratory phases. Three cases were treated with three to seven intravenous injections of $5 \mathrm{c.cm}$. gluco-calcium (Lilly), given at the height of the febrile attacks, and these resulted in an almost immediate abatement of the pyrexia and the intestinal and muscular symptoms. Experimental trials of this method have not yet been made, though it is possible that such treatment is not lethal to Trichinella, but acts more in a detoxifying and perhaps nutritive and corrective manner: its effect during the acute stages suggests such a mode of action, and it is unlikely that it has any similarity to the calcium therapy outlined previously. A more extensive clinical and experimental trial of this method is urged.

\section{Summary}

The clinical and symptomatic treatment of trichinosis is briefly discussed and measures for the destruction of the parasite in the host are reviewed. Radium irradiation and eatharsis appear to be of no practical value. The use of convalescent antiserum to control the toxic features of the disease should be tried if such serum could be made readily available. 
Most chemotherapeutic measures involving the use of anthelmintics, antiseptics, disinfectants and related substances, organic arsenicals and similar substances, and certain miscellaneous drugs have been shown experimentally to be of no value and perhaps dangerous. There is experimental evidence, however, that the subcutaneous injection in olive oil or gum arabic of a suspension of the commercial thymol preparation carvasept or the oral administration of butolan (Bayer) (carbamic acid ester of p-oxy-diphenyl-methane) is lethal to a certain proportion of reproductive adults in rats, and their use results in a lessening in severity of the muscle invasion: a clinical trial of butolan should be made.

Excellent results have followed the intravenous administration of $5 \mathrm{c.cm}$. of gluco-calcium (Lilly) during the hitherto intractable acute migratory phase; this form of therapy should be given further clinical and experimental trials.

Experiments with rats, rabbits, and cats have shown that calcification after encystment may be considerably hastened by the administration of some vitamin-D-containing substance, supplemented by a supply of calcium in the form of a bland organic salt, thus protecting the host from toxic effects following encystment. Though a warning has been given that this treatment may result in a dangerous metastatic calcification of other organs, a further judicious clinical trial should be made. It should be noted, however, that this treatment is of no value before encystment of the larvae-that is, before four to five weeks have elapsed after infection.

\section{REFERENCES}

Africa, C., and Lucker, J. T. (1931). Proc. Soc. exp. Biol., N.Y., 28,432 .

Algora y Nieto, M. (1924). Siglo méd., 73, 160. Abstract in J. Amer. med. Ass., 1924, 82, 1232

Anon. (1935). "Calcification Acceleration of Trichina Cysts." N. Amer. Vet., 16 (3), 24.

Augustine, D. L., and Theiler, H. (1932). Parasitology, 24, 60.

Bachman, G. W., and González, J. O. (1936). Proc. Soc. exp. Biol., N.Y., 35, 215.

Banerii, N. (1933). Amer. J. med. Sci., 186, 689.

Baudet, E. A. R. F. (1931). Arch. Schiffs- u. Tropen-Hyg., 35

(1935). Tijdschr. Diergeneesk.. 62, 527.

Booth, B. A., Goerhing, W. N., and Kahn, M. (1916). J. Amer. med. Ass., 67, 2000

Bugge, G. (1934). Arch. wiss. prakt. Tierheilk., 68, 24.

Caronia, G. (1937). Arch. ital. Sci. med. colon., 18, 283.

Chopra, R. N., and Chandler, A. C. (1928). Anthelmintics and their Uses, Baillière, Tindall and Cox, London.

Chükri, H. (1930). Klin. Wschr., 9, 298.

Clark, A. J. (1929). Applied Pharmacology, J. and A. Churchill, London. Craig, C. F., and Faust, E. C. (1937). Clinical Parasitology,

Culbertson, J. T., and Kaplan, S. S. (1937). J. Parasit., 23, 560

Culbertson, (1938). Parasitology, 30,156

Fantus, B. (1935). J. Amer. med. Ass., 104, 472.

Faust, E. C. (1929). Proc. Soc. exp. Biol., N.Y., 26, 748

Ferenbaugh, T. L., Segal, L., and Schulze, H. A. (1938). J. Amer. med. Ass. 110,1434

Fleischmann, P. (1930). Dtsch. med. Wschr., 56, 648.

Greenwood, D. N. (1935). J. Chemother., 12, 232.

Grove, J. S. (1925) J. Amer. med. Ass., 85, 349.

Gruber, G. B. (1926). Ergebn. Hyg. Bakt., 8, 165.

Gruber, G. B. (1926). Ergebn. Hyg. Bakt..

Hall, M. C., and Wigdor, M. (1918). Arch. intern. Med., 22, 601

Hardy, T. L. (1927). Lancet, $2,1380$.

Hassin, G. B., and Diamond, I. B. (1926). Arch. Neurol. Psychiat. Chicago, 15, 34.

Herzberg, E., and Vitenson, I. (1930). British Medical Journal, 1 650

Kahn, M. (1917). N.Y. med. J., 105, 1137.

McCoy, O. R. (1931). Amer. J. Hyg., 14, 484.

(1932). Science, 85, 364

(1935). Amer. J. Hyg., 21, 200

(1938). Proc. Soc. exp. Biol., N.Y., 38, 461

McDonald, E. P., and Waddell, K. C. (1929). J. Amer. med. Ass. 92449 .

McNerthney, J. B., and McNerthney, W. B. (1916). Ibid., 67, 1086

Martin, L. D. (1936). Med. Países. calidos., 11, 75.
Maternowska, L. (1933). Zbl. Bakt., Abt. 1, Orig. 129 (3/4),

Matoff, K. (1937). Tierärztl. Rdsch., 43, (21) 354, (22) 369

Miller, T. J., jun., McCoy, O. R., and Bradford, W. L. (1932). J. Amer. med. Ass., 98, 1242

Munk, F. (1917). Med. Klinik, 13, 16, 161, 217.

Pierce, G. N., jun., and McNaught, J. B. (1937). Proc. Soc. exp. Biol., N.Y.., 36, 579

Ragany, J. (1935). Med. Rec. N.Y., 142, 335

Roth, H. (1938). J. Parasit., 24, 220.

Salzer, B. F. (1916). J. Amer. med. Ass., 67, 579.

- (1917). Med. Rec. N.Y., 81, 261.

Schulemann, W. (1920). Dtsch. med.Wschr., 46, 1050.

Schwartz, B. (1917). J. Amer. med. Ass., 69, 884.

Seifert, O. (1929). Handbuch der pathogenen Mikroorganismen 6, 995, Kolle and Wassermann.

Sogemeier, E. (1938). Miinch. med. Wschr., 85, 669

Spink, W. W. (1934). Arch. intern. Med., 54, 805.

Sprehn, C. (1931). Tierärztl. Rdsch., 5, 77.

Trawinski, A. (1935). Zbl. Bakt., Abt. 1, Orig. 134 (3/4), 145.

Tyzzer, E. E., and Honiej, J. A. (1917). J. Parasit., 3, 43.

Van Cott, J., M., and Lintz, W. (1914). J. Amer. med. Ass., 62 680.

van Someren, V. D. (1937). British Medical Journal, 2, 1162. (1938). In preparation.

von Brand, Th., Holtz, F., and Putschar, W. (1932). Arch. exp. Path. Pharmak., 167, 113.

- and Vogel, H. (1933). Z. Parasitenk., 6, 308.

- Otto, G. F., and Abrams, E. (1937). J. Parasit., 23, 533

Otto, G. F., and A38). Amer. J. Hyg., 27, 461

Wantland, W. W. (1934). Proc. Soc. exp. Biol., N.Y., 32, 438 (1935). J. Parasit., 21, 441.

(1936). Ibid., 22, 537, 538

(1938). Ibid., 24, 167

Weltmann, O. (1931). Med. Klinik, 27, 1600.

Wenderoth, A. E. P. (1917). Inaug. Diss., Kiel.

\section{BRONCHOSCOPY IN PULMONARY TUBERCULOSIS}

BY

H. V. MORLOCK, M.C., M.D., M.R.C.P.

Physician to the London Chest Hospital and to the Miller and Hampstead General Hospitals

AND

E. H. HUDSON, M.B., M.R.C.P.

Assistant Physician to the London Chest Hospital and to the West London Hospital

The value of bronchoscopy in the diagnosis and treatment of non-tuberculous diseases of the lungs is well established, but its use in tuberculous disease is still in the experimental stage. That this is so is due to the fear that such an investigation might do more harm than good to a phthisical patient. The same fear delayed the use of lipiodol investigation in these patients, but it has proved to be without foundation, and both these methods are now being employed freely in the investigation of the tuberculous patient.

\section{Cases indicating Use of Bronchoscopy}

It is not suggested that a diagnostic bronchoscopy should be a routine investigation in all cases; nevertheless, the recent recognition of tracheo-bronchial tuberculosis (a condition which can only be diagnosed by bronchoscopic vision) demonstrates that the more frequent use of this method is justified. We have endeavoured to employ it only in those cases in which consideration of the synıptoms, physical signs, and a skiagram has led us to belis:ve that it would definitely provide further useful information. These cases have fallen into the following groups: (1) sputum-negative cases; (2) those showing areas of collapse ; (3) those showing areas of obstructive emphysema ; (4) those with symptoms suggestive of tracheo-bronchial tuberculosis ; (5) those in which another pulmonary disease is associated with pulmonary tuberculosis. 